\title{
Monitoring Cortico-cortical Evoked Potentials using Only Two 6- strand Strip Electrodes for Gliomas Extending to the Dominant Side of Frontal Operculum During One-step Tumor Removal Surgery
}

Taiichi Saito ( $\nabla$ taiichis@gmail.com )

Tokyo Joshi lka Daigaku https://orcid.org/0000-0002-5016-9619

Yoshihiro Muragaki

Tokyo Women's Medical University

Manabu Tamura

Tokyo Women's Medical University

Takashi Maruyama

Tokyo Women's Medical University

Masayuki Nitta

Tokyo Women's Medical University

Shunsuke Tsuzuki

Tokyo Women's Medical University

Atsushi Fukui

Tokyo Women's Medical University

Shunichi Koriyama

Tokyo Women's Medical University

Takakazu Kawamata

Tokyo Women's Medical University

Research Article

Keywords: glioma, cortico-cortical evoked potentials, awake craniotomy, language function, frontal operculum

Posted Date: September 20th, 2021

DOI: https://doi.org/10.21203/rs.3.rs-883872/v1

License: (9) This work is licensed under a Creative Commons Attribution 4.0 International License. Read Full License 


\section{Abstract}

Resection of the dominant side of gliomas extending to the frontal operculum has a high-risk of permanent language dysfunction. Here, we report recording cortico-cortical evoked potentials (CCEP) using only two 6-strand strip electrodes to monitor languagerelated fibers intraoperatively. We examined whether this simple procedure is useful for removing gliomas extending to the dominant side of frontal operculum. This study included five cases of glioma extending to the left frontal operculum. The frontal language area (FLA) was first identified by functional mapping during awake craniotomy. Next, a 6-strand strip electrode was placed just above the FLA, while on the temporal lobe side, an electrode was placed so as to slide parallel to the Sylvian fissure toward the posterior language area. Electrical stimulation was performed using the electrode on the frontal side, and CCEPs were measured from the electrode on the temporal side. CCEPs were detected in all cases. Immediately after surgery, all patients demonstrated language dysfunction to varying degrees. CCEP decreased up to $10 \%$ in one patient, who recovered language function after 24 months. CCEP decreased slightly up to $80 \%$ in one, and in the three other cases, CCEPs did not change. These four patients soon recovered language function within 2 weeks to 1 month. This study confirmed the utility of CCEP monitoring using only two 6-strand strip electrodes during one-step surgery. We believe this simple method helped in monitoring intraoperative language function and aided predicting its postoperative recovery, in patients with gliomas extending to the dominant side of frontal operculum.

\section{Introduction}

Maximum resection of the dominant side of gliomas extending to the frontal operculum poses a high risk of permanent language dysfunction. To prevent this, injury of the frontal language area (FLA) should be avoided, as well as that of language-related subcortical fibers. Direct brain functional mapping using electrical stimulation during awake craniotomy is the most efficient method to identify language-related cortical and subcortical areas.[2] However, verbal responses during awake craniotomies are subjective and they depend on the consciousness level and cooperativeness of the patient. Additionally, it is difficult to determine whether postoperative speech impairments will improve based solely on the degree of speech symptoms observed during surgery. In recent years, using cortico-cortical evoked potentials (CCEP) has attracted attention as an intraoperative evaluation method for the optimal functioning of intercortical networks including those involving language.[12,23,7,20,22,8] We have previously reported that the application of the CCEP procedure for the intraoperative monitoring of language-related fibers may be useful during the removal of glioma near brain regions involved in language processing.[18] We showed that CCEP monitoring during glioma resection can be helpful for the objective assessment of intraoperative language function and for predicting the prognosis of postoperative language functional recovery.

In the previous study,[18] we performed CCEP measurements during the removal of gliomas in two steps, as follows: (1) functional mapping and CCEP measurements were performed before tumor resection using chronic subdural grid electrodes placed in the first surgery, and (2) in the second operation, the grid electrodes were replaced with 6-strand strip electrodes in order to monitor intraoperative CCEP during tumor resection. However, performing two-step surgeries can increase the risk of infection and are exhausting for the patient. Furthermore, in other prior reports of tumor resection combined with CCEP monitoring, when grid electrodes were used in the procedure,[22,20,13,23,19,12] it was thought that the electrodes interfered with the removal of tumors, especially those extending to the dominant side of frontal operculum.

Therefore, in the present investigation, we omitted step (1), and after performing functional mapping during awake craniotomy, CCEP was measured intraoperatively during a one-step tumor removal intervention, solely by using two 6-strand strip electrodes. We hoped that, if successful, our simple method of CCEP monitoring for assessing language function during brain tumor resection could become widespread in the future. Then, in this study, we examined whether CCEP monitoring using only two 6-strand strip electrodes is useful for removing gliomas extending to the dominant side of frontal operculum.

\section{Patients And Methods}

\section{Patient population and preoperative evaluation}

From January 2018 to March 2021, awake craniotomies were performed in 102 patients with supratentorial glioma at the Tokyo Women's Medical University. Intraoperative CCEP monitoring using 6-strand strip electrodes during awake craniotomy was performed in five patients (all men; mean age 48 years) with glioma extending to the dominant side of frontal operculum. For these patients, a 
preoperative evaluation of tumor localization was performed using magnetic resonance imaging (MRI), which included T2-weighted, fluid attenuated inversion recovery (FLAIR), and pre- and postcontrast T1-weighted images. From MR images, we established whether the tumors extended to the dominant side of frontal operculum (part of the inferior frontal gyrus). Which side was the dominant hemisphere of language function was evaluated using functional MRI. The study protocol was approved by the Ethics Committee of the Tokyo Women's Medical University. Because of the retrospective nature of the design, the institutional review board waived the requirement for informed consent. To ensure patient privacy, we omitted identifiers from the current results.

\section{Surgery}

Surgery was performed according to previously published concept of brain tumor removal, which specified aiming for maximal resection of the tumor whilst minimizing the risk of permanent language disorder.[10,11,15-18] Intraoperative MRI (AIRIS IIM; Hitachi Medical Corporation, Tokyo, Japan) and updated-neuronavigation were performed routinely. These procedures aided the tumor resection by providing information about accurate tumor localization. Surgery generally aimed to maximal possible resection of the enhanced area with contrast medium on T1-weighted MR images for tumors suspected to be glioblastomas and the hyperintense area on T2-weighted or FLAIR images for tumors suspected to be grade II or III gliomas. Pathological diagnosis was performed based on criteria from the 2016 World Health Organization classification of tumors of the central nervous system.[6]

\section{Intraoperative language functional mapping}

We performed language functional mapping according to the method we have previously published,[16,18] as follows: electrical cortical stimulations were applied with repetitive square-wave biphasic currents of alternating polarity (pulse width, $0.2 \mathrm{~ms}$; frequency, $50 \mathrm{~Hz}$; duration, 1-2 s) using an Ojemann cortical bipolar stimulator (OCS-1; Integra Radionics, Burlington, MA). A continuous digital electrocorticogram was monitored to detect seizures and after-discharges. Stimulus intensity was increased steadily from $2 \mathrm{~mA}$ using stepwise increments of $1 \mathrm{~mA}$ until an effect was attained or abnormalities on the electrocorticogram (ECOG) were recognized. The maximum stimulus intensity was $6 \mathrm{~mA}$ (biphasic current; $12 \mathrm{~mA}$ ). As in the previously published method, $[16,18]$ frontal language-related areas were defined when stimulation consistently interrupted, or slowed the ability of the patient to name a pictured object, pronounce a familiar written Japanese word, and generate an action verb during an image presentation of a task, without seizures, after-discharges, or positive or negative motor responses of the tongue and facial muscles. Removal of the tumor was accompanied by subcortical stimulation through the cavity wall of the resection, with the purpose of identifying subcortical language pathways. The devices used for this procedure and the parameters of stimulation, including intensity parameters, were similar to those used for cortical mapping. During the entire procedure, while the patient was awake, constant observation of the patient's spontaneous speech ability was maintained through continuous conversation.

\section{Intraoperative CCEP monitoring}

In all cases, the left frontotemporal craniotomy was performed so that the frontal operculum was widely exposed, and the anterior half of the superior temporal gyrus was revealed on the temporal lobe side. The FLA was first identified by functional mapping during awake craniotomy in all cases. Next, a 6-strand strip electrode (diameter $3 \mathrm{~mm}$, distance between centers $10 \mathrm{~mm}$, Unique Medical Co.) was placed just above the FLA. On the temporal lobe, another 6-strand strip electrode was placed to slide parallel to the Sylvian fissure, from the surgical field toward the posterior superior temporal gyrus (STG), where the posterior language area (PLA) is anatomically presumed (Fig. 1). As in the previous study,[18] two adjacent electrodes were stimulated in a bipolar fashion with a constant-current square wave of alternating polarity (pulse width $0.3 \mathrm{~ms}$, frequency $1 \mathrm{~Hz}$ ). Continuous digital ECoG activity was recorded to identify seizures and after-discharges. The stimulus intensity increased steadily from $2 \mathrm{~mA}$ (biphasic current; $4 \mathrm{~mA}$ ), using stepwise increments of $2 \mathrm{~mA}$ until the CCEP response was attained or ECoG abnormalities were noted. Actual stimulus intensities from all five patients in the present study were $6 \mathrm{~mA}$ (biphasic current; $12 \mathrm{~mA}$ ). The reference electrode was placed in the area of the contralateral mastoid process.

The bandpass filter for data acquisition was set at $5-1500 \mathrm{~Hz}$ with a sampling rate of $5000 \mathrm{~Hz}$ for each channel. In all cases, electrical stimulation was performed through the electrode placed on the frontal lobe, and CCEPs were measured from the electrode on the temporal lobe. We looked for CCEP to monitor defined as the highest observed negative peaks in the waveform amplitudes obtained after stimulation. During each stimulation session, we averaged two or more trials of 100 responses. We excluded the first $10 \mathrm{~ms}$ from the stimulus onset from each response, in order to avoid artifacts caused by the stimulation. A complete CCEP was obtained after 100 seconds, but it usually stabilized after the application of 30 stimuli; therefore, the changes, if present, could be 
identified in 30-40 seconds. All stimulations and CCEP recordings were performed with a dedicated multimodal neuromonitor (Neuromaster Mee-2000, Nihon Kohden Co.). Patients were not specifically requested to perform any task during CCEP measurement when they were awake.

\section{Postoperative evaluation}

The extent of resection was assessed with intraoperative MRI performed after tumor removal. We calculated the extent of resection (EOR) using the contrast-enhanced area in enhanced tumors, and using hyperintense areas on T2-weighted images in non-enhanced tumors. The initial evaluation of speech function was performed after the patient awoke from anesthesia and subsequently on a daily basis until discharge from the hospital. Thereafter, all patients followed up regularly with the neurosurgeon, in the outpatient clinic of our hospital.

\section{Results}

\section{Summary of demographics and clinical data}

Clinical characteristics of the five patients are summarized in Table 1. Mean age of the study population was 48 years (range, 38-63 years). All tumors were on the left side and extended to the frontal operculum. The pathological diagnoses for the tumors were WHO grade III gliomas in four of the five patients and one patient had a glioblastoma, IDH wild-type. The mean EOR was 93\% (range, $85 \%$ $98 \%)$ for all patients.

\section{Intraoperative CCEP findings and language function}

Intraoperative CCEP findings and language functions of the five patients are summarized in Table 2. In all five patients, the FLA was successfully identified with intraoperative language functional mapping during awake craniotomy. The stimulus intensities of all patients were $6 \mathrm{~mA}$ (biphasic current; $12 \mathrm{~mA}$ ). In all patients, the best responses of CCEP were obtained from the electrode placed on the PLA during stimulation of the electrode on the FLA. Bimodal CCEP peaks were observed in three of five patients $($ cases $1,4,5)$, while in the other two patients (cases 2,3), only a monomodal peak was observed. During tumor resection, the CCEP monitoring did not change in three patients, decreased up to $10 \%$ in one, and decreased up to $80 \%$ in another patient.

Only one patient had mild preoperative dysarthria, while the other patients presented no speech disturbances preoperatively. For patients in cases 1 and 2, changes in CCEPs appeared during removal of the deep part of the tumor, while language dysfunctions were also being observed. Although the CCEPs of the other three patients did not change during surgery, these patients also showed mild paraphasia probably due to pressure on the frontal language-related area during the surgical procedure.

\section{Postoperative course}

Soon after the operation, all patients showed speech disturbances to varying degrees. In case 1, the patient presented severe disturbance of naming a object and repetition error. While the other four patients showed mild paraphasia, their ability to repeat words was preserved. Language function promptly recovered in four patients after operation (range 0.5-1 months) (Table 2). However, the time to speech recovery in case 1 (the patient's CCEPs decreased to $10 \%$ of their initial amplitudes) was longer (24 months) than in the other cases.

\section{Representative cases}

\section{Case 1}

A 41-year-old man presented with mild dysarthria. He was identified with a tumor located in the left middle and inferior frontal gyri, as well as the precentral gyrus, which extended to the frontal operculum, based on MRI findings. The tumor had a hypointense signal and showed no contrast enhancement with contrast medium on T1-weighted images, but presented a hyperintense signal on T2weighted images (Fig. 2A). The tumor resection was performed during an awake craniotomy. Intraoperative electrical functional mapping identified the FLA on the pars triangularis. Then, a 6-strand strip electrode was placed just above the FLA (Fig. 1). On the side of the temporal lobe, a 6-strand strip electrode was placed so as to slide parallel to the Sylvian fissure from the surgical field toward the posterior STG which contained the PLA (Fig. 1). Continuous CCEP monitoring was performed with stimulation of the FLA 
and recording from the PLA. During the removal of the deep part of the tumor, we noted failure of naming a picture accompanied by a $40 \%$ decrease in CCEP amplitudes (Fig. 2B). Furthermore, during the resection of the deep posterior part of the tumor, the patient suddenly showed severe repetition failure as well as a $90 \%$ decrease in CCEP amplitudes (Fig. 2B). Consequently, the resection was terminated. The patient presented severe language dysfunction immediately after surgery. Postoperative MRI revealed an $85 \%$ resection of the tumor (Fig. 2C). Histopathological diagnosis findings revealed that the tumor was an anaplastic oligodendroglioma. The patient's language dysfunction was long-lasting, taking 24 months to recover.

Case 2

A 38-year-old man presented with partial epilepsy. His language abilities were normal preoperatively. MRI showed that his tumor was located in the left precentral gyrus and inferior frontal gyrus, extending to the frontal operculum. The tumor showed a hypointense signal and no enhancement as revealed by medium contrast T1-weighted images, and a hyperintense signal on T2-weighted images (Fig. 3A). Intraoperative functional mapping identified the FLA on the anterior side of the pars opercularis. A 6-strand strip electrode was placed just above the FLA (Fig. 3B). On the side of the temporal lobe, a 6-strand strip electrode was placed on the PLA, toward the posterior STG (Fig. 3B). Continuous CCEP monitoring was performed with stimulation from the FLA electrode and monitoring from the PLA electrode. CCEPs were mostly stable throughout tumor removal (Fig. 3C). However, during resection of the deep posterior part of the tumor, mild dysarthria and paraphasia were noticed, alongside a 20\% decrease in CCEP amplitudes (Fig. 3C). The patient also exhibited mild dysarthria and paraphasia immediately after surgery. A postoperative MRI revealed that $95 \%$ of the tumor was removed (Fig. 3D). The histopathological diagnosis was anaplastic oligodendroglioma. His language dysfunction recovered within a month.

Case 4

An intracranial tumor was incidentally discovered in a 63-year-old man. He had no neurological symptoms, and his preoperative speech function was normal. MRI showed that the tumor was mainly located in the middle and inferior frontal gyri and extended to the frontal operculum. The tumor showed a hypointense signal and no enhancement on medium contrast T1-weighted images, and a hyperintense signal on T2-weighted images (Fig. 4A). Electrical stimulations for intraoperative functional mapping identified the FLA on the posterior part of the middle frontal gyrus. A 6-strand strip electrode was placed just above the FLA (Fig. 4B). On the temporal lobe side, a 6-strand strip electrode was placed toward the posterior STG, where the PLA was usually found (Fig. 4B). The CCEP monitoring was continuously performed with stimulation from the FLA and recording from the PLA. During tumor removal, the two modal CCEPs were stable and did not decrease (Fig. 4C). However, mild paraphasia appeared intraoperatively, probably due to pressure on the frontal language-related area associated with the surgical procedure. The patient showed mild paraphasia immediately after surgery. Postoperative MRI revealed that $90 \%$ of the tumor was removed (Fig. 4D). The histopathological diagnosis confirmed an anaplastic oligodendroglioma. The patient's language impairment recovered within a month.

\section{Discussion}

In this study, intraoperative CCEP monitoring using only two 6-strand strip electrodes also correlated well with intraoperative language function and the time to recovery of language impairments in patients with gliomas extending to the left frontal operculum as previous reports.[18,22,23] The extensive removal of the dominant side of such tumors posed a high risk of severe language functional deterioration. However, by using our method of intraoperative CCEP monitoring during awake craniotomies, we accomplished a mean EOR of $93 \%$ for tumors located in brain areas responsible for language processing. This suggests that this simple surgical method increased the safety of removing tumors which extend to the dominant side of frontal operculum. Furthermore, since strip electrodes are smaller than grid electrodes, they did not interfere with the procedure of tumor removal, likely contributing to an increase in EORs. The present results support the utility of our simple method of CCEP monitoring with one-step surgery and using only two 6-strand strip electrodes, instead of grid electrodes as previously reported.[22,20,13,23,19,12]

\section{Validity of our method of CCEP monitoring in this study}

In prior studies, the FLA is relatively localized, whereas the PLA is reported to have a diffuse extent (Fig. 5A). [9,23] Enatsu et al. [5] reported patients' PLAs were found in various places, including the areas anterior to the junction of the Rolandic and the Sylvian fissures, as well as the angular gyrus and the temporo-occipital junction. When CCEP measurements were performed using only 6strand strip electrodes, it was important to identify the FLA with certainty and place the electrode directly above it (Fig. 5B). On the

Page 5/14 
other hand, the PLA is thought to be spread diffusely, so the CCEP from the PLA can be obtained solely by placing the electrode in its approximate location (Fig. 5B). Regarding the direction of electrical stimulation to obtain the response of CCEP, Matsumoto et al. [9] suggested that because of the scattered distribution of neurons in the PLA projecting to the FLA, only a part of the PLA can be activated neurons in the FLA at one time by the stimulation technique in their study. While, in the FLA, projection fibers to the PLA are more densely present (Fig. 5A). Therefore, in order to activate the projection fibers related to language function more efficiently with a single stimulus, to stimulate the FLA and detect CCEPs in the PLA can be more effective method as in the current study (Fig. 5B).

\section{CCEP findings and language recovery}

Previous studies have reported that decreases in CCEP amplitudes suggest the presence of injuries to language-related subcortical fibers.[23,22,18] In the current study, the CCEP of the patient in case 1 showed suddenly a $90 \%$ decrease in amplitude, suggesting that language-related subcortical fibers were severely injured intraoperatively. The patient in case 2 showed a $20 \%$ decrease in CCEP amplitudes, indicating mild injury to language-related subcortical fibers. The CCEPs of the patients in the other three cases did not change intraoperatively, suggesting that language-related subcortical fibers were preserved. Immediately after surgery, all patients showed language dysfunctions to varying degrees, including the three patients with unchanged CCEPs. They presented mild paraphasia both during and after the operation, probably due to pressure on the frontal language-related area associated with the surgical procedure.

In terms of language functional recovery, this took between $0.5-1$ month in four out of five patients. The time to speech recovery of the patient in case 1 , whose language-related fibers were presumably severely damaged during surgery, was evidently longer ( 24 months) than that of the other patients. However, language function is known to successfully recover even after the removal of tumors insuring critical speech areas, due to neuroplasticity.[4,1,14] On the other hand, the plasticity of the frontal operculum (ventral premotor cortex) is known to be limited by its subcortical connections with language-related areas.[3,21] This highlights the importance of preserving language-related subcortical fibers in order to ensure language functional reorganization following damage during resection for gliomas extending to the frontal operculum. Consequently, CCEP monitoring is useful to ensure the utmost preservation of language-related fibers during tumor removal and language functional recovery due to neuroplasticity.

\section{Limitations}

The present study has its limitations. While pre- and postoperative tractography using diffusion tensor imaging is useful in the evaluation of the degree of injury to language-related subcortical fibers, this study did not investigate how tractography results were related to CCEP changes. Another limitation includes the retrospective nature of the design, as well as the small population sample. Therefore, our observations need to be reproduced and validated by future larger prospective studies which contain more thorough neurofunctional imaging analyses.

\section{Conclusion}

This study validated the utility of the CCEP monitoring using only two 6-strand strip electrodes during a one-step surgery: we confirm that this is a reliable method of assessing language function intraoperatively and it helps predicting the time to recovery of speech impairments postoperatively, in patients with gliomas extending to the dominant side of frontal operculum. The current method is also useful for monitoring the injury of language-related subcortical fibers in order to preserve them. For the success of this simple procedure, we emphasize the importance of precisely identifying the FLA, in the sense that the stimulating electrode needs to be placed exactly above this area.

\section{Declarations}

\section{Funding}

This study was supported by the Japan Society for the Promotion of Science (JSPS) KAKENHI Grant No. 21 K09138.

\section{Conflicts of interest/Competing interests}

The authors declare no potential conflicts of interest. 
Availability of data and material

The datasets during and/or analyzed during the current study available from the corresponding author on reasonable request.

\section{Code availability}

Not applicable.

\section{Ethics approval}

Ethical approval was obtained from the ethics committee at Tokyo Women's Medical University (No. 1955-R3).

\section{Consent to participate}

Given the retrospective design, the institutional review board waived the requirement for informed consent. We took the opt-out method. To protect patient privacy, we removed all identifiers from our records upon completion of our analyses.

\section{Consent for publication}

Not applicable.

\section{Authors' contributions}

Conceptualization: [Taiichi Saito], [Yoshihiro Muragaki]; Formal analysis and investigation: [Manabu Tamura], [Takashi Maruyama]; Writing - original draft preparation: [Taiichi Saito]; Writing - review and editing: [Yoshihiro Muragaki], [Taiichi Saito], [Takakazu Kawamata]; Funding acquisition: [Taiichi Saito]; Resources: [Masayuki Nitta], [Shunsuke Tsuzuki], [Atsushi Fukui]; Visualization: [Shunsuke Tsuzuki], [Atsushi Fukui]; Supervision: [Takakazu Kawamata].

\section{Acknowledgements}

Special thanks are devoted to Dr. Takashi Komori, Ms. Soko Ikuta, Asuka Komori and Mr. Takashi Sakayori for valuable help with clinical work and data analysis. We would like to thank Editage (www.editage.com) for English language editing.

\section{References}

1. Benzagmout M, Gatignol P, Duffau H (2007) Resection of World Health Organization Grade Il gliomas involving Broca's area: methodological and functional considerations. Neurosurgery 61:741-752; discussion 752-743. doi:10.1227/01.NEU.0000298902.69473.77

2. Byrne RW, Sanai N, Landeiro JA, Duffau H (2018) Introduction: Advances in intraoperative brain mapping. Neurosurg Focus 45:Intro. doi:10.3171/2018.10.FocusVid.Intro

3. Duffau H (2014) The huge plastic potential of adult brain and the role of connectomics: new insights provided by serial mappings in glioma surgery. Cortex 58:325-337. doi:10.1016/j.cortex.2013.08.005

4. Duffau H, Capelle L, Denvil D, Sichez N, Gatignol P, Lopes M, Mitchell MC, Sichez JP, Van Effenterre R (2003) Functional recovery after surgical resection of low grade gliomas in eloquent brain: hypothesis of brain compensation. $\mathrm{J}$ Neurol Neurosurg Psychiatry 74:901-907. doi:10.1136/jnnp.74.7.901

5. Enatsu R, Kubota Y, Kakisaka Y, Bulacio J, Piao Z, O'Connor T, Horning K, Mosher J, Burgess RC, Bingaman W, Nair DR (2013) Reorganization of posterior language area in temporal lobe epilepsy: a cortico-cortical evoked potential study. Epilepsy Res 103:73-82. doi:10.1016/j.eplepsyres.2012.07.008

6. Louis DN, Perry A, Reifenberger G, von Deimling A, Figarella-Branger D, Cavenee WK, Ohgaki H, Wiestler OD, Kleihues P, Ellison DW (2016) The 2016 World Health Organization Classification of Tumors of the Central Nervous System: a summary. Acta Neuropathol 131:803-820. doi:10.1007/s00401-016-1545-1

7. Matsumoto R, Kunieda T, Nair D (2017) Single pulse electrical stimulation to probe functional and pathological connectivity in epilepsy. Seizure 44:27-36. doi:10.1016/j.seizure.2016.11.003 
8. Matsumoto R, Nair DR, Ikeda A, Fumuro T, Lapresto E, Mikuni N, Bingaman W, Miyamoto S, Fukuyama H, Takahashi R, Najm I, Shibasaki H, Luders HO (2012) Parieto-frontal network in humans studied by cortico-cortical evoked potential. Hum Brain Mapp 33:2856-2872. doi:10.1002/hbm.21407

9. Matsumoto R, Nair DR, LaPresto E, Najm I, Bingaman W, Shibasaki H, Luders HO (2004) Functional connectivity in the human language system: a cortico-cortical evoked potential study. Brain 127:2316-2330. doi:10.1093/brain/awh246

10. Muragaki Y, Iseki H, Maruyama T, Kawamata T, Yamane F, Nakamura R, Kubo O, Takakura K, Hori T (2006) Usefulness of intraoperative magnetic resonance imaging for glioma surgery. Acta Neurochir Suppl 98:67-75. doi:10.1007/978-3-211-333037_10

11. Muragaki Y, Iseki H, Maruyama T, Tanaka M, Shinohara C, Suzuki T, Yoshimitsu K, Ikuta S, Hayashi M, Chernov M, Hori T, Okada Y, Takakura K (2011) Information-guided surgical management of gliomas using low-field-strength intraoperative MRI. Acta Neurochir Suppl 109:67-72. doi:10.1007/978-3-211-99651-5_11

12. Nakae T, Matsumoto R, Kunieda T, Arakawa Y, Kobayashi K, Shimotake A, Yamao Y, Kikuchi T, Aso T, Matsuhashi M, Yoshida K, Ikeda A, Takahashi R, Lambon Ralph MA, Miyamoto S (2020) Connectivity Gradient in the Human Left Inferior Frontal Gyrus: Intraoperative Cortico-Cortical Evoked Potential Study. Cereb Cortex 30:4633-4650. doi:10.1093/cercor/bhaa065

13. Ookawa S, Enatsu R, Kanno A, Ochi S, Akiyama Y, Kobayashi T, Yamao Y, Kikuchi T, Matsumoto R, Kunieda T, Mikuni N (2017) Frontal Fibers Connecting the Superior Frontal Gyrus to Broca Area: A Corticocortical Evoked Potential Study. World Neurosurg 107:239-248. doi:10.1016/j.wneu.2017.07.166

14. Robles SG, Gatignol P, Lehericy S, Duffau H (2008) Long-term brain plasticity allowing a multistage surgical approach to World Health Organization Grade II gliomas in eloquent areas. J Neurosurg 109:615-624. doi:10.3171/JNS/2008/109/10/0615

15. Saito T, Muragaki Y, Maruyama T, Tamura M, Nitta M, Okada Y (2015) Intraoperative Functional Mapping and Monitoring during Glioma Surgery. Neurol Med Chir (Tokyo) 55 Suppl 1:1-13

16. Saito T, Muragaki Y, Maruyama T, Tamura M, Nitta M, Tsuzuki S, Konishi Y, Kamata K, Kinno R, Sakai KL, Iseki H, Kawamata T (2016) Difficulty in identification of the frontal language area in patients with dominant frontal gliomas that involve the pars triangularis. J Neurosurg 125:803-811. doi:10.3171/2015.8.JNS151204

17. Saito T, Tamura M, Chernov MF, Ikuta S, Muragaki Y, Maruyama T (2018) Neurophysiological Monitoring and Awake Craniotomy for Resection of Intracranial Gliomas. Prog Neurol Surg 30:117-158. doi:10.1159/000464387

18. Saito T, Tamura M, Muragaki Y, Maruyama T, Kubota Y, Fukuchi S, Nitta M, Chernov M, Okamoto S, Sugiyama K, Kurisu K, Sakai $\mathrm{KL}$, Okada Y, Iseki H (2014) Intraoperative cortico-cortical evoked potentials for the evaluation of language function during brain tumor resection: initial experience with 13 cases. J Neurosurg 121:827-838. doi:10.3171/2014.4.JNS131195

19. Suzuki Y, Enatsu R, Kanno A, Yokoyama R, Suzuki H, Tachibana S, Akiyama Y, Mikami T, Ochi S, Yamakage M, Mikuni N (2019) The Influence of Anesthesia on Corticocortical Evoked Potential Monitoring Network Between Frontal and Temporoparietal Cortices. World Neurosurg 123:e685-e692. doi:10.1016/j.wneu.2018.11.253

20. Tamura Y, Ogawa H, Kapeller C, Prueckl R, Takeuchi F, Anei R, Ritaccio A, Guger C, Kamada K (2016) Passive language mapping combining real-time oscillation analysis with cortico-cortical evoked potentials for awake craniotomy. J Neurosurg 125:15801588. doi:10.3171/2015.4.JNS15193

21. van Geemen K, Herbet G, Moritz-Gasser S, Duffau H (2014) Limited plastic potential of the left ventral premotor cortex in speech articulation: evidence from intraoperative awake mapping in glioma patients. Hum Brain Mapp 35:1587-1596. doi:10.1002/hbm. 22275

22. Yamao Y, Matsumoto R, Kunieda T, Arakawa Y, Kobayashi K, Usami K, Shibata S, Kikuchi T, Sawamoto N, Mikuni N, Ikeda A, Fukuyama H, Miyamoto S (2014) Intraoperative dorsal language network mapping by using single-pulse electrical stimulation. Hum Brain Mapp 35:4345-4361. doi:10.1002/hbm.22479

23. Yamao Y, Suzuki K, Kunieda T, Matsumoto R, Arakawa Y, Nakae T, Nishida S, Inano R, Shibata S, Shimotake A, Kikuchi T, Sawamoto N, Mikuni N, Ikeda A, Fukuyama H, Miyamoto S (2017) Clinical impact of intraoperative CCEP monitoring in evaluating the dorsal language white matter pathway. Hum Brain Mapp 38:1977-1991. doi:10.1002/hbm.23498

\section{Tables}




\begin{tabular}{|c|c|c|c|c|c|c|}
\hline Case No. & Age & Sex & Side of lesion & Tumor location & Tumor histology & $\%$ Lesion Resection \\
\hline 1 & 41 & M & Lt & MFG + PreCG + IFG & Anaplastic oligodendroglioma & 85 \\
\hline 2 & 38 & M & $\mathrm{Lt}$ & PreCG + IFG & Anaplastic oligodendroglioma & 95 \\
\hline 3 & 51 & M & Lt & PreCG + Insula + IFG & Anaplastic astrocytoma, IDH mutant & 98 \\
\hline 4 & 63 & M & Lt & MFG + IFG & Anaplastic oligodendroglioma & 90 \\
\hline 5 & 48 & M & Lt & MFG + PreCG + IFG & Glioblastoma, IDH wild-type & 95 \\
\hline
\end{tabular}

Table 2 Summary of intraoperative CCEP findings and progress of language function

\begin{tabular}{|c|c|c|c|c|c|c|c|c|c|}
\hline \multirow[b]{2}{*}{$\begin{array}{l}\text { Case } \\
\text { No. }\end{array}$} & \multirow[b]{2}{*}{ Anesthesia } & \multicolumn{4}{|c|}{ Intraoperative CCEP } & \multicolumn{4}{|c|}{ Language Function } \\
\hline & & $\begin{array}{l}\text { Stimulus } \\
\text { Intensity } \\
(\mathrm{mA})\end{array}$ & $\begin{array}{l}\text { Peak } \\
\text { Latency } \\
\text { (msec) }\end{array}$ & $\begin{array}{l}\text { Direction of } \\
\text { Stimulation }\end{array}$ & $\begin{array}{l}\text { Changes } \\
\text { During } \\
\text { Tumor } \\
\text { Removal }\end{array}$ & Preop & Intraope & $\begin{array}{l}\text { Postope } \\
\text { disturbance }\end{array}$ & $\begin{array}{l}\text { Time to } \\
\text { Recovery } \\
\text { (mos) }\end{array}$ \\
\hline 1 & $\begin{array}{l}\text { local } \\
\text { (awake) }\end{array}$ & 6 & 22,81 & $\mathrm{FLA} \rightarrow \mathrm{PLA}$ & $\begin{array}{l}\text { decreased } \\
\text { (up to } \\
10 \% \text { ) }\end{array}$ & $\begin{array}{l}\text { mild } \\
\text { dysarthria }\end{array}$ & $\begin{array}{l}\text { naming } \\
\text { failure, } \\
\text { repetition } \\
\text { failure }\end{array}$ & yes & 24 \\
\hline 2 & $\begin{array}{l}\text { local } \\
\text { (awake) }\end{array}$ & 6 & 78 & $\mathrm{FLA} \rightarrow \mathrm{PLA}$ & $\begin{array}{l}\text { decreased } \\
\text { (up to } \\
80 \% \text { ) }\end{array}$ & normal & $\begin{array}{l}\text { mild } \\
\text { dysarthria, } \\
\text { mild } \\
\text { paraphasia }\end{array}$ & yes & 1 \\
\hline 3 & $\begin{array}{l}\text { local } \\
\text { (awake) }\end{array}$ & 6 & 55 & $\mathrm{FLA} \rightarrow \mathrm{PLA}$ & unchanged & normal & $\begin{array}{l}\text { mild } \\
\text { paraphasia }\end{array}$ & yes & 0.5 \\
\hline 4 & $\begin{array}{l}\text { local } \\
\text { (awake) }\end{array}$ & 6 & 28,102 & $\mathrm{FLA} \rightarrow \mathrm{PLA}$ & unchanged & normal & $\begin{array}{l}\text { mild } \\
\text { paraphasia }\end{array}$ & yes & 1 \\
\hline 5 & $\begin{array}{l}\text { local } \\
\text { (awake) }\end{array}$ & 6 & 26,108 & $\mathrm{FLA} \rightarrow \mathrm{PLA}$ & unchanged & normal & $\begin{array}{l}\text { mild } \\
\text { paraphasia }\end{array}$ & yes & 1 \\
\hline
\end{tabular}

\section{Figures}




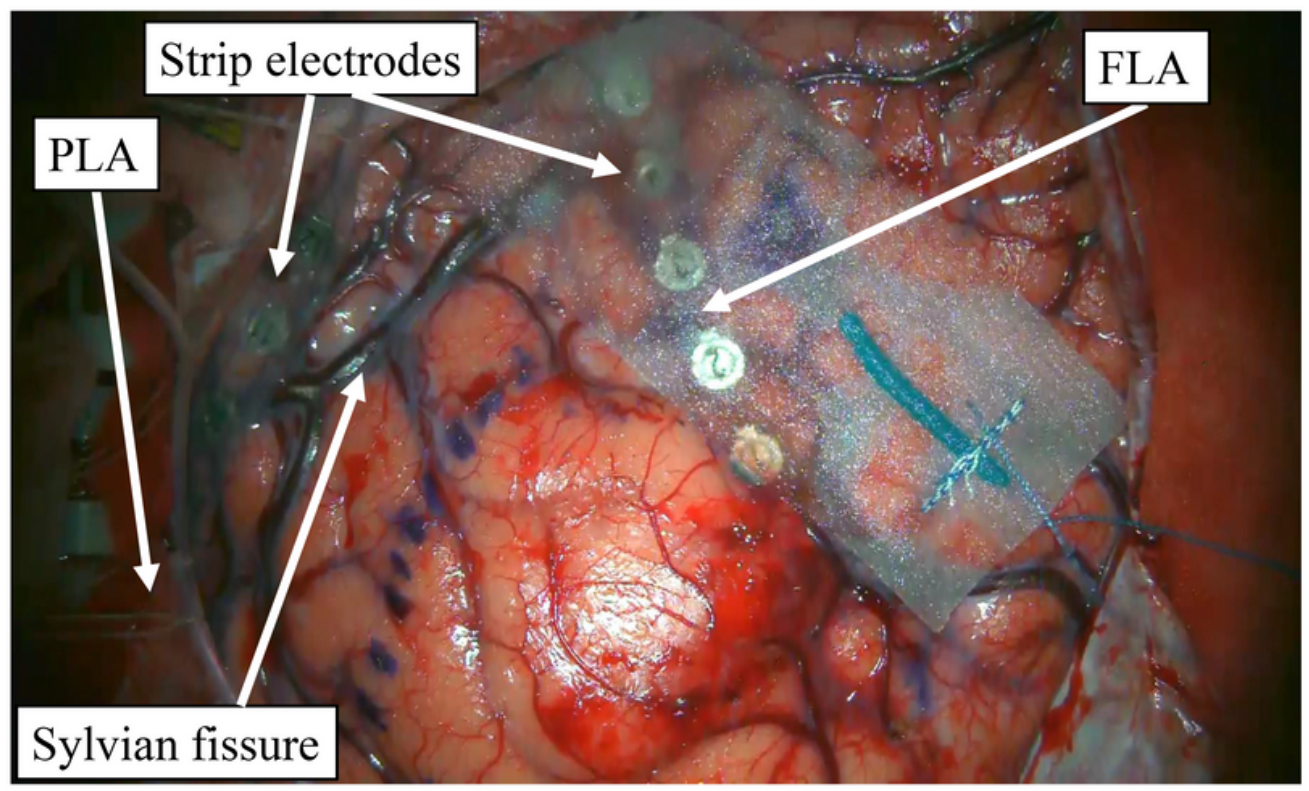

Figure 1

CCEP monitoring using only 6-strand strip electrodes. The frontal language area (FLA) was first identified by functional mapping during awake craniotomy. Next, a 6-strand strip electrode was placed just above the FLA, while on the temporal lobe, a 6-strand strip electrode was placed so as to slide parallel to the Sylvian fissure and toward the posterior superior temporal gyrus where the posterior language area is usually located (PLA). 

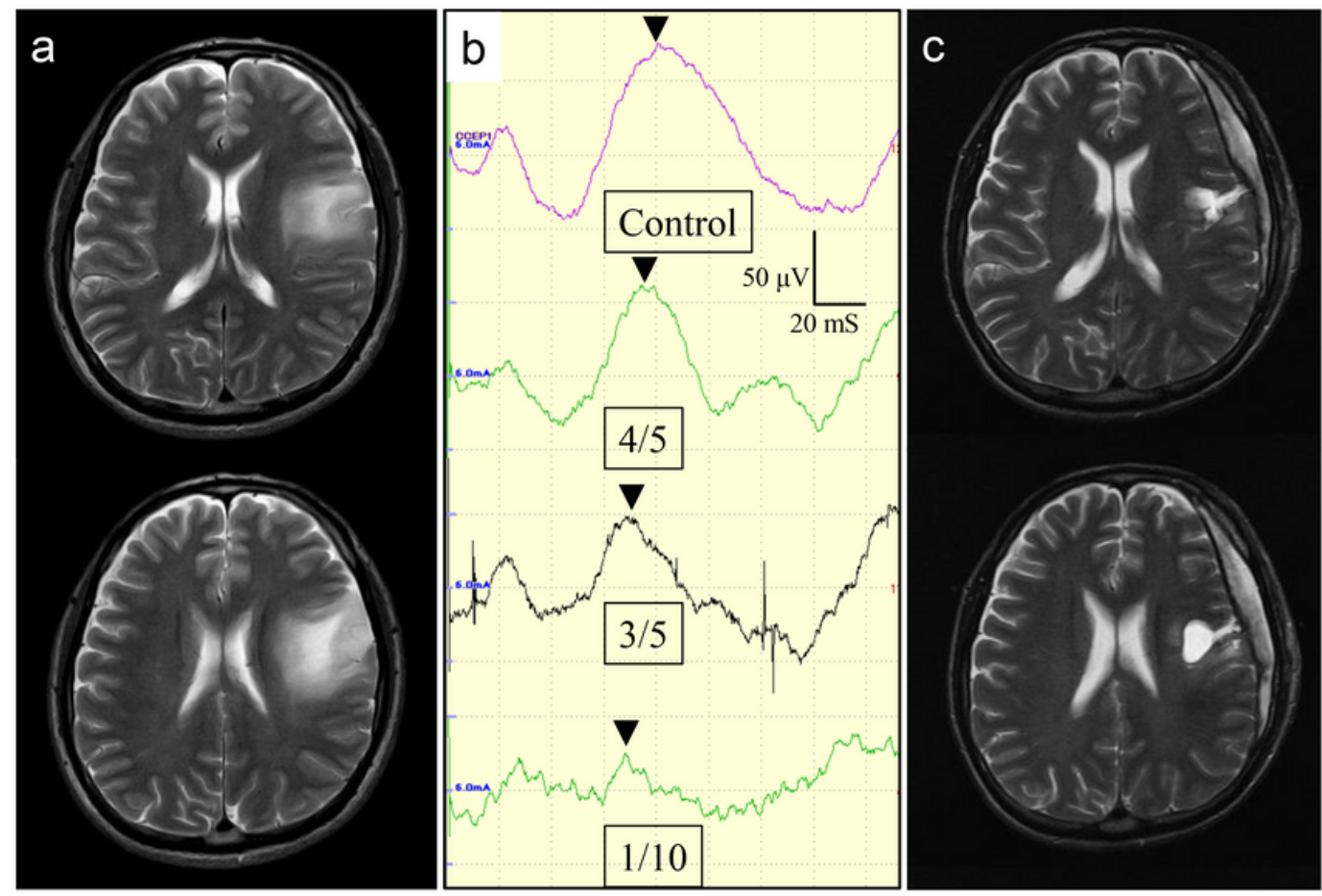

Figure 2

Case 1. Anaplastic oligodendroglioma in a 41-year-old man. Preoperative T2-weighted MR images (a) revealed a tumor with hyperintense signal in the left middle and inferior frontal gyri, as well as in the precentral gyrus extending to the frontal operculum. CCEP monitoring demonstrated a decrease in amplitude, of up to $10 \%$ (b). Postoperative T2-weighted MR images (c) revealed that $85 \%$ of the tumor was removed. 

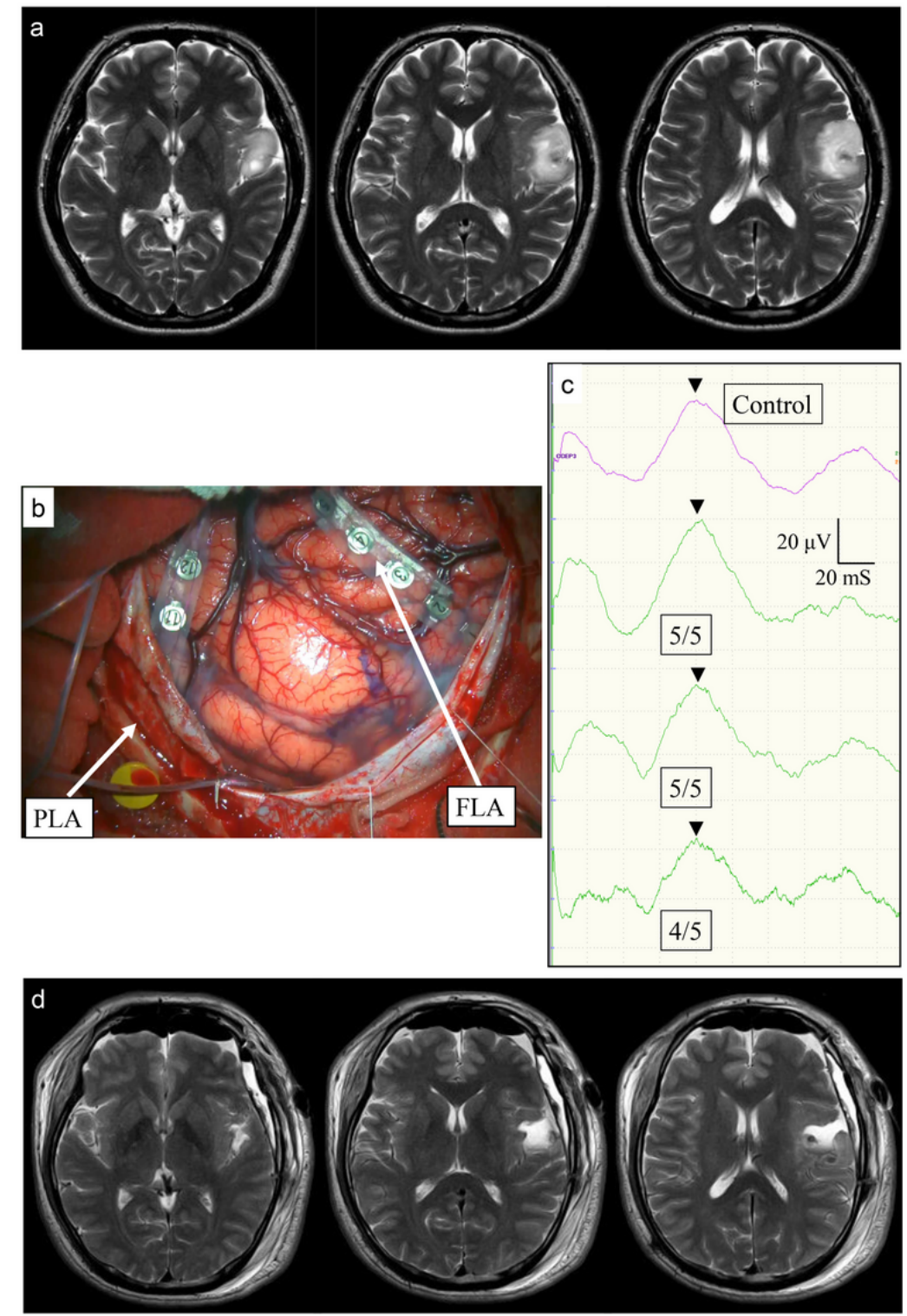

Figure 3

Case 2. Anaplastic oligodendroglioma in a 38-year-old man. Preoperative T2-weighted MR images (a) revealed a tumor with hyperintense signal in the left precentral gyrus and inferior frontal gyrus extending to the frontal operculum. A 6-strand strip electrode was placed just above the FLA (b). On the temporal lobe side, a 6-strand strip electrode was placed toward the PLA (b). CCEP monitoring showed a gradual decrease of up to $80 \%$ (c). Postoperative T2-weighted MR images (d) showed that $95 \%$ of the tumor was removed. 

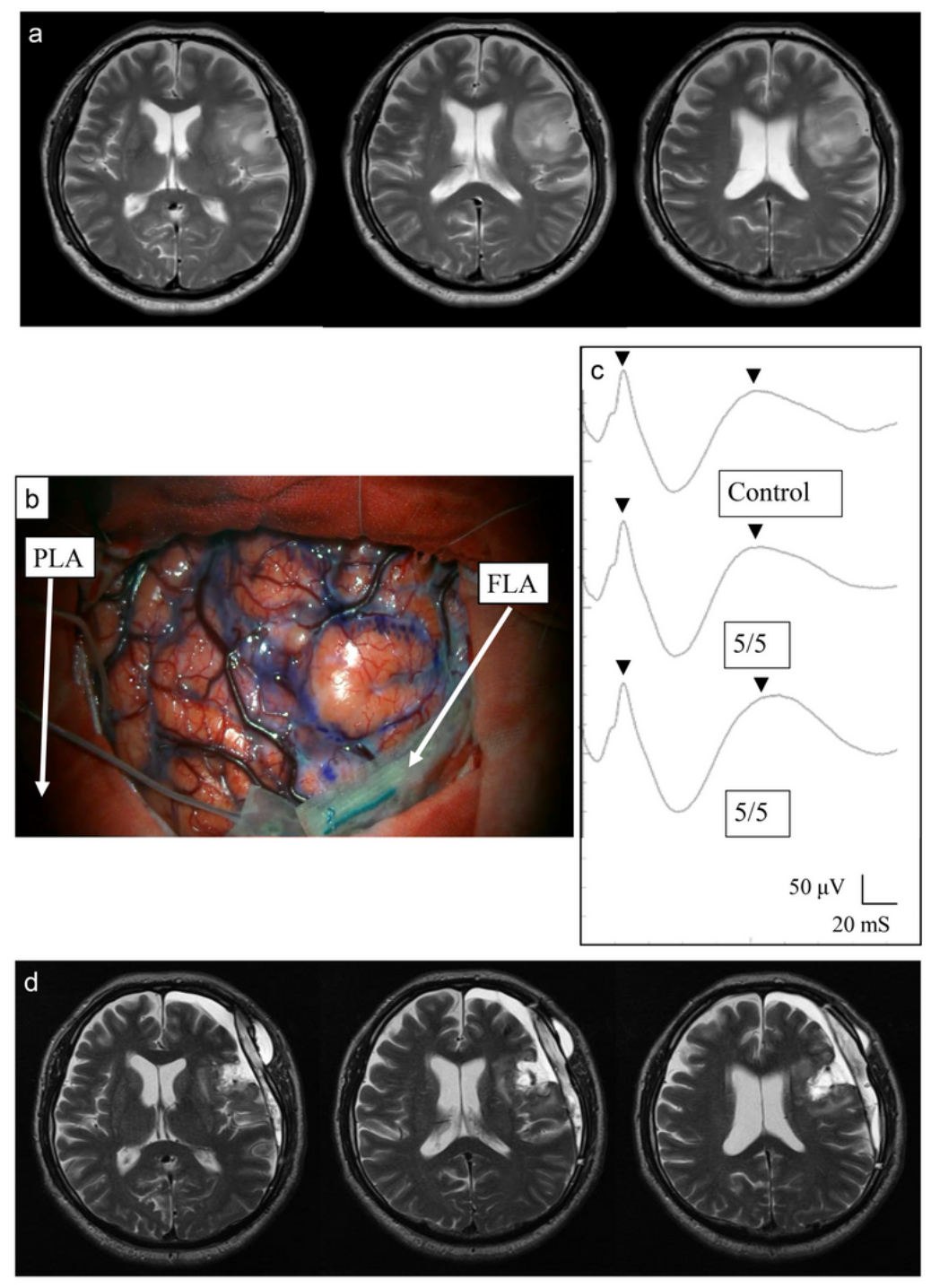

Figure 4

Case 4. Anaplastic oligodendroglioma in a 63-year-old man. Preoperative T2-weighted MR images (a) revealed a tumor with hyperintense signal in the middle and inferior frontal gyri extending to the frontal operculum. A 6-strand strip electrode was placed just above the FLA (b). On the temporal lobe side, a 6 -strand strip electrode was placed toward the PLA (b). CCEP monitoring showed stable evoked potential amplitudes (c). Postoperative T2-weighted MR images (d) showed that $90 \%$ of the tumor was removed. 

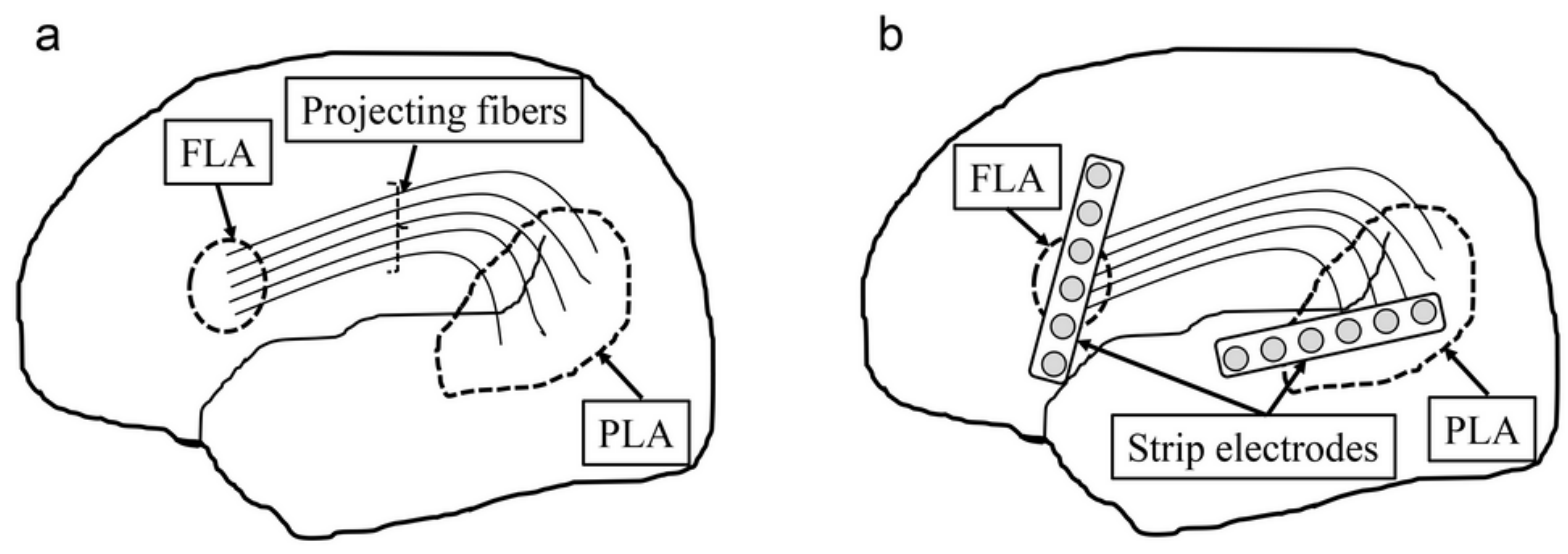

\section{Figure 5}

(a) The frontal language area (FLA) is relatively localized, whereas the posterior language area (PLA) is reported to have a diffuse extent. Language-related projection fibers in the FLA are more densely present than in the PLA (b) When CCEP measurements are performed using only 6-strand strip electrodes, it is important to identify the FLA with certainty and place the electrode directly above it to activate the projection fibers related to language function more efficiently with a single stimulus. On the other hand, it is considered that on the PLA, CCEP can be obtained solely by placing the electrode in the approximate location of this brain region. 